nigra et al through concentric bipolar needle electrodes. Football shaped lesions were made bilaterally at brain stem or lenticular nucleus, which ranged between 2.0 and $2.5 \mathrm{~mm}$ in maximum diameter. Twenty one days in average survival period were allowed for the Metrazol injection after the lesions were made.

1. Epilepsy elicitee by intravenous administration of Metrazol was apparently of subcortical origin both in the normal control and the lesion groups, when spike or sharp wave is indicated as an epileptic discharge.

2. There was a certain parallel relationship, without discrepancy, in the thresholds between the origination of epileptic discharge and major seizure.

3. The lesioning of the bilateral lenticular nuclei tended to increase the threshold of major seizure. It appeared specifically obtained when the dorsal portion of the putamen was lesioned.

4. A characteristic increment of the major seizure threshold was obtained when a portion of the brain stem from prerubral area or the Forel-H field of the subthalamic region to postrubral area or upper mesencephalic reticular formation including red nucleus was partially lesioned. The increment was more marked than the one obtained at the same sized lesioning of the lenticular nucleus.

5. The portion of the brain stem mentioned above seems to appear a certain functional unit for maintaing an epileptic excitability at subcortical level and also for controlling its progression toward major seizure.

\title{
14. Influence of VL Simulotion upon Motoneuron Activity
}

\author{
Keiichi Murata \\ Department of Physiology, Nagoya University School of Medicine \\ Kenichiro SUGITA and Terushige Dor \\ Department of Surgery, Nagoya University School of Medicine
}

The effect of stereotaxic stimulation of the Nucleus ventralis lateralis thalami on the activity of motoneurons was studied on inmobilized cats using Flaxedil and Nembutal narcosis.

More than 300 points in the thalamus were stimulated three-dimensionally in arrays of 0.5 or $1.0 \mathrm{~mm}$ distance by one of 6 electrodes mounted in parallel, separated by $1 \mathrm{~mm}$ distance and inserted stereotaxically into the thalamus. Repetitive stimuli of $50-500 \mathrm{cps}, 0.05-1.0 \mathrm{msec}$ and $0.5-2.0 \mathrm{~V}$ 
were fed between one of these 6 electrodes and an indifferent electrode attached to the incised skin.

The amplitudes of evoked potentials elicited by a single shock to the dorsal column and recorded from VP through the 6 electrode array were plotted on an atlas of the thalamus and the error of relative position of VL due to individual differences to the scales of stereotaxic apparatus was corrected. The positions of stimulating electrode tips were verified histologically afterwards.

A laminectomy was performed from $L_{6}$ to $S_{2}$ levels, to record the monosynaptic reflex as well as the spontaneous discharges of single motoneurons. Single shock or low frequency stimulation of less than $50 \mathrm{cps}$ to VL did not exert any influence upon them. During volleys of several hundred cps, the monosynaptic reflex recorded from some portions of a ventral root and the spontaneous activity of some motoneurons were inhibited, while the monosynaptic reflex recorded from other portions and the spontaneous activity of some other motoneurons were enhanced.

The same method was applied at the entrance of a muscle nerve to its corresponding muscle in the hind limbs as at the spinal cord. In most preparations repetitive stimuli of VL inhibited the monosynaptic reflex and the spontaneous motoneuron activity in extensor (M. gastrocnemius, M. soleus, etc.), while flexor (M. tibialis anterior, M. peroneus longs, M. biceps femoris, M. semitendinosus, etc.) motoneuons were activated during such stimulation.

The stimulation of VP always caused inhibition of motoneuron activity and after cessation it was followed by the complete inhibition of monosynaptic reflex and of motoneuron activity sometimes for over $30 \mathrm{sec}$. In some cats the motoneurons were activated during $\mathrm{CM}$ stimulation and inhibited after cessation of the stimulus like after cessation of VL stimulation.

Recording electromyographically the motor unit activity from limb muscles of cats without Flaxedil and of human patients during stereotaxic surgical operations, the reciprocal effect of VL stimulation is not so clear as in cats with Flaxedil. This could be partly due to participation of the closed $\gamma$ system in the motoneuron activity.

\title{
15. Effect of Stereotaxic Surgery on Cerebral Palsy
}

\author{
Hoshio Katsumata \\ Dept. of Orthopedic Surgery, Kyoto Prefectural Medical College

